\title{
Minimal Resolutions of Lattice Ideals and Integer Linear Programming
}

\author{
Emilio Briales-Morales, Antonio Campillo-López \\ Pilar Pisón-Casares and Alberto Vigneron-Tenorio
}

\begin{abstract}
A combinatorial description of the minimal free resolution of a lattice ideal allows us to the connection of Integer Linear Programming and Algebra. The non null reduced homology spaces of some simplicial complexes are the key. The extremal rays of the associated cone reduce the number of variables.
\end{abstract}

\section{Introduction}

The objective of this paper is to describe how Integer Linear Programming allows us to obtain the minimal free resolution of a lattice ideal, $I$, from the generators of the semigroup, $S$, which parametrizes the associated algebraic variety.

Concretely, Hilbert bases of some diophantine systems are employed. These bases are the solution of the typical Integer Linear Programming Problem, but the minimality with respect to a cost map is not imposed. Recall that this typical problem is:

$$
\min \left\{c \cdot x \quad \mid A x=b, x \in \mathbb{N}^{n}\right\},
$$

where $A$ is an integer matrix, $b$ an integer vector and $c$ a real vector $(c \cdot x$ is the cost map).

2000 Mathematics Subject Classification: Primary 13D02, 14M25; Secondary 13P10, 68W30, 90C27.

Keywords: Resolutions, simplicial complex, syzygy, lattice ideal, regularity, Integer Linear Programming, Hilbert bases, Gröbner bases. 
Anybody who has solved linear diophantine equations in non negative integers, even with the more recent methods (see [18], [20], [23], [44] and [46]), knows that only in the case of a few variables the problem is tractable. It is well-known that this problem is NP-complete (see for example [37]). Therefore, from the computational viewpoint, our description is not practical in order to obtain the minimal free resolution. However, the method can be used to the contrary. Our description allows the understanding of the relation between the syzygies of the ideal and Integer Linear Programming. One can compute with Gröbner bases using for example the Schreyer Theorem and its improvements (see [33]), and look for applications to Integer Programming. This philosophy comes from [19] and [46], and provides a lot of applications in [50]. For example, the typical Integer Linear Programming Problem can be solved computing the reduced Gröbner basis of an associated lattice ideal. Or for instance, the Graver basis ([28]) of an ideal can be obtained from a reduced Gröbner basis of its Lawrence lifting, which is its unique minimal generating set. Nevertheless, at the moment this philosophy has only been employed in the case of the ideal $I$, but not the syzygies of the higher order (the ideal can be considered as the syzygies of order zero). Our description yields the generalization.

As in [30] and [48], the combinatorial objects we use are simplicial complexes. Concretely, for any element of the semigroup $S$, we associate two simplicial complexes. The elements in the semigroup represent the degrees of the syzygies, in fact, the minimal free resolution is $S$-graded. The study of the non null reduced homology spaces of the simplicial complexes provides the concept of $i$-triangulation. This concept is the key in order to understand the relation between Integer Linear Programming and Algebra, concretely, between Hilbert bases and $i$ th syzygies.

By means of a partition of the generating set of $S$, the number of variables is reduced to the number of extremal rays of the associated cone. This is another possible point to continue researching. A generator over each extremal ray is chosen. Fixing the attention on this subset of generators, a new resolution is considered, the minimal free resolution of $I$ over a polynomial ring with only the variables corresponding to these generators.

We begin in section 1 with the definition of the algebraic objects we employ. In section 2 we give the combinatorial description of the two minimal free resolutions. Section 3 is dedicated to the $i$-triangulations in a simplicial complex and some applications. The exposition of how to compute both resolutions with Gröbner bases is in section 4. All these sections include the results we have already obtained using the techniques this paper describes. For details the reader may also want to consult the reference joined to the concrete result. 
Another possible application of our description is in Toric Geometry. Normal toric varieties [26], and more generally, non-normal toric varieties [27] and [50], appear as algebraic varieties whose ideals are lattice ones. Among our results can be found descriptions of the regularity of these ideals as well as upper bounds for the degree of their generators. It is expected that there is some relation between these results and the conjetures of [24] and [50] (see also [49] and [39]). For a survey of the modern developments in the theory of toric varieties see [21]. Some applications of this theory to the Arithmetic and Integer Programming can be found in [17].

The hull resolution is another free resolution of a lattice ideal. This resolution is a generalization of the results for generic lattice ideals in [2] and [38]. It was introduced in [5] using Integer Programming. The study of the minimality of this resolution is a current research objective (see [3] where the case of unimodular lattice ideals is considered, and [36] for the monomial curves in the affine space of low dimension).

On the other hand, it is known that any binomial ideal is an intersection of cellular ideals [25]. The cellular ideals are closely related to the lattice ideals. Using cellular decomposition of a binomial ideal, it is possible to obtain information about the binomial ideal from the properties of the lattice ideals (for example, primary decomposition or nilpotence index, see [34] and $[35])$.

\section{The two minimal free resolutions associated with a lattice ideal}

Let $k$ be a commutative field and $k[\mathbf{X}]=k\left[X_{1}, \ldots, X_{n}\right]$ the polynomial ring in $n$ indeterminates, and the ideal $\mathfrak{m}=\left(X_{1}, \ldots, X_{n}\right)$.

Let $\mathcal{L} \subset \mathbb{Z}^{n}$ be a lattice. The ideal of the lattice $\mathcal{L}$ is

$$
I_{\mathcal{L}}=\left\langle\mathbf{X}^{u^{+}}-\mathbf{X}^{u^{-}} \mid \mathbf{u} \in \mathcal{L}\right\rangle,
$$

where $u=u^{+}-u^{-}, u^{+}, u^{-} \in \mathbb{N}^{n}$, have disjoint support.

Let $S$ be a cancellative commutative semigroup, with zero element and generated by $n$ elements $\Lambda=\left\{m_{1}, \ldots, m_{n}\right\}$. Thus, $S$ is a subsemigroup of a finitely generated abelian group. Denote $G(S)$ the smallest group containing $S$. The semigroup $k$-algebra is $k[S]=\bigoplus_{m \in S} k \chi^{m},\left(\chi^{m} \cdot \chi^{m^{\prime}}=\chi^{m+m^{\prime}}\right)$. The ideal of $S$ relative to $\Lambda$ is $\operatorname{ker}\left(\varphi_{0}\right)$, where $\varphi_{0}$ is the $k$-algebra morphism

$$
\varphi_{0}: k[\mathbf{X}] \longrightarrow k[S]
$$

defined by $\varphi_{0}\left(X_{i}\right)=\chi^{m_{i}}$. Notice that $\varphi_{0}$ is surjective, and hence $k[S] \simeq$ $k[\mathbf{X}] / \operatorname{ker}\left(\varphi_{0}\right)$. 
If $I_{\mathcal{L}}$ is the ideal of the lattice $\mathcal{L} \subset \mathbb{Z}^{n}$, then $I_{\mathcal{L}}$ is the ideal of the subsemigroup of $\mathbb{Z}^{n} / \mathcal{L}$ generated by $\left\{e_{1}+\mathcal{L}, \ldots, e_{n}+\mathcal{L}\right\}$, where the $e_{i}$ 's are the unit vectors.

On the other hand, the ideal of any semigroup $S$ relative to a generating set $\Lambda$ is the ideal of the lattice $\left\{u=\left(u_{1}, \ldots, u_{n}\right) \in \mathbb{Z}^{n} \mid \sum u_{i} m_{i}=0\right\}$ (see [52] for details).

From now on, we fix a lattice $\mathcal{L}$ or equivalently a semigroup $S$. Assume that $\mathcal{L} \cap \mathbb{N}^{n}=(0)$, or equivalently $S \cap(-S)=(0)$. Let $I$ be the ideal relative to the generating set $\Lambda=\left\{m_{1}, \ldots, m_{n}\right\}$ of $S$. Notice that $I$ is $S$-graded because $\varphi_{0}$ is an $S$-graded morphism of degree zero, considering $k[S]$ with the natural $S$-grading and $k[\mathbf{X}]$ as an $S$-graded ring, assigning the degree $m_{i}$ to $X_{i}$. The condition $S \cap(-S)=(0)$ says that $k[\mathbf{X}]_{m}$, the homogeneous elements of degree $m \in S$ in $k[\mathbf{X}]$, is a $k$-vector space of finite dimension (see [8]).

Another application of the condition $S \cap(-S)=(0)$, is Nakayama's lemma for $S$-graded $k[\mathbf{X}]$-modules (see [8]). Thus, there exists an $S$-graded minimal free resolution of $k[S]$, which is unique up to isomorphism. We denote such a resolution by

$$
0 \rightarrow k[\mathbf{X}]^{b_{p}} \stackrel{\varphi_{p}}{\longrightarrow} \cdots \rightarrow k[\mathbf{X}]^{b_{2}} \stackrel{\varphi_{2}}{\longrightarrow} k[\mathbf{X}]^{b_{1}} \stackrel{\varphi_{1}}{\longrightarrow} k[\mathbf{X}] \stackrel{\varphi_{0}}{\longrightarrow} k[S] \rightarrow 0,
$$

and let $N_{i}=\operatorname{ker}\left(\varphi_{i}\right)$ be the $i$ th module of syzygies $0 \leq i \leq p\left(N_{0}=I\right)$.

Notice that

$$
b_{i+1}=\operatorname{dim}\left(N_{i} / \mathfrak{m} N_{i}\right),
$$

where $N_{i} / \mathfrak{m} N_{i}$ is considered as a $k$-vector space. Moreover, since this space is $S$-graded, if $V_{i}(m):=\left(N_{i} / \mathfrak{m} N_{i}\right)_{m}$, where $m \in S$, then

$$
b_{i+1}=\sum_{m \in S} \operatorname{dim} V_{i}(m)
$$

The Auslander-Buchbaum theorem guarantees that

$$
p=n-\operatorname{depth}_{k[\mathbf{X}]} k[S],
$$

where $\operatorname{depth}_{k[\mathbf{X}]} k[S]$ is the depth of $k[S]$ as $k[\mathbf{X}]$-module. It is known that $\operatorname{depth}_{k[\mathbf{X}]} k[S]$ is bounded by $\operatorname{dim} k[S]$, which is the rank of the abelian group $G(S)$. In the case the bound is reached, $k[S]$ is a Cohen-Macaulay ring. Thus, this case will be called Cohen-Macaulay case. On the other hand, if $S \neq\{0\}$, it is satisfied that $\operatorname{depth}_{k[\mathbf{X}]} k[S] \geq 1$.

Assume that $\operatorname{rank}(G(S))=d$, let $V=G(S) \bigotimes_{\mathbb{Z}} \mathbb{Q}$, and let $C(S)$ be the cone generated by the image $\bar{S}$, of $S$ in $V$. The cone $C(S)$ is strongly convex because $S \cap(-S)=(0)$. Thus, if $f$ is the number of extremal rays of $C(S)$, then $f \geq d$. This implies that there exists a set $E \subset \Lambda$ with $\sharp E=f$, such that $C(E)=C(S)$, where $C(E)$ is the cone in $V$ generated by $E$. Fix such a set $E$. 
The Apery set $Q$ of $S$ relative to $E$ is defined as

$$
Q=\{q \in S \mid q-e \notin S, \forall e \in E\} .
$$

Denote $k[E]$ the subalgebra of $k[S]$,

$$
k[E]=\bigoplus_{m \in S_{E}} k \chi^{m}
$$

where $S_{E}$ is the subsemigroup of $S$ generated by $E$. Let $k\left[\mathbf{X}_{E}\right]$ be the polynomial ring in the $f$ indeterminates associated with $E . k\left[\mathbf{X}_{E}\right]$ can be projected over $k[E]$, it is enough to associate to the indeterminate $X_{i}$ the symbol $\chi^{m_{i}}$, for any $m_{i} \in E . k[S]$ is a $k[E]$-module, and therefore also a $k\left[\mathbf{X}_{E}\right]$-module. The set

$$
\left\{\chi^{q} \mid q \in Q\right\}
$$

is a minimal system of generators of $k[S]$ as $k[E]$-module, and therefore, also as $k\left[\mathbf{X}_{E}\right]$-module. Since $k[E] \subset k[S]$ is an integral extension and $k[S]$ is finitely generated as a $k[E]$-algebra, $k[S]$ is finitely generated as a $k[E]$ module. So $k[S]$ is a finitely generated $k[E]$-module, and $Q$ is a finite set. Suppose that $\beta_{0}=\sharp Q, Q=\left\{q_{1}, \ldots, q_{\beta_{0}}\right\}$, and consider

$$
\Phi_{0}: k\left[\mathbf{X}_{E}\right]^{\beta_{0}} \longrightarrow k[S]
$$

defined by $\Phi_{0}\left(e_{i}\right)=\chi^{q_{i}}, 1 \leq i \leq \beta_{0}$. We can consider the $S$-graded minimal resolution of $k[S]$ as $k\left[\mathbf{X}_{E}\right]$-module

$$
0 \rightarrow k\left[\mathbf{X}_{E}\right]^{\beta_{q}} \stackrel{\Phi_{q}}{\longrightarrow} \cdots \rightarrow k\left[\mathbf{X}_{E}\right]^{\beta_{2}} \stackrel{\Phi_{2}}{\longrightarrow} k\left[\mathbf{X}_{E}\right]^{\beta_{1}} \stackrel{\Phi_{1}}{\longrightarrow} k\left[\mathbf{X}_{E}\right]^{\beta_{0}} \stackrel{\Phi_{0}}{\longrightarrow} k[S] \rightarrow 0
$$

which is unique except isomorphisms. Let $M_{i}=\operatorname{ker}\left(\Phi_{i}\right)$ be the $i$ th module of syzygies of $k[S]$ as $k\left[\mathbf{X}_{E}\right]$-module, $0 \leq i \leq q$. As before, by $S$-graded Nakayama's lemma, we obtain

$$
\beta_{i+1}=\sum_{m \in S} \operatorname{dim} W_{i}(m)
$$

where $W_{i}(m):=\left(M_{i} / \mathfrak{m}_{E} M_{i}\right)_{m}$ is considered as a $k$-vector space, and $\mathfrak{m}_{E}$ is the ideal of $k\left[\mathbf{X}_{E}\right]$ generated by the indeterminates of $\mathbf{X}_{E}\left(X_{i}\right.$ such that $\left.m_{i} \in E\right)$.

The Auslander-Buchbaum theorem guarantees that $q=f-\operatorname{depth}_{k\left[\mathbf{x}_{E}\right]} k[S]$, where $\operatorname{depth}_{k\left[\mathbf{X}_{E}\right]} k[S]$ is the depth of $k[S]$ as $k\left[\mathbf{X}_{E}\right]$-module. Using the combinatorial descriptions of the above two resolutions in the following section, and the theorem 4.1 in [13], one gets that

$$
\operatorname{depth}_{k[\mathbf{x}]} k[S]=\operatorname{depth}_{k\left[\mathbf{x}_{E}\right]} k[S] .
$$

Therefore, $p \geq q$.

Now, we will call the $S$-graded minimal free resolution of $k[S]$ as $k[\mathbf{X}]$ module the long resolution, and the short resolution the $S$-graded minimal free resolution of $k[S]$ as $k\left[\mathbf{X}_{E}\right]$-module. 


\section{Combinatorial description of the resolutions}

Assume that $S \neq(0)$, and consider the $S$-graded minimal free resolution,

$$
0 \rightarrow k[\mathbf{X}]^{b_{p}} \stackrel{\varphi_{p}}{\longrightarrow} \cdots \rightarrow k[\mathbf{X}]^{b_{2}} \stackrel{\varphi_{2}}{\longrightarrow} k[\mathbf{X}]^{b_{1}} \stackrel{\varphi_{1}}{\longrightarrow} k[\mathbf{X}] \stackrel{\varphi_{0}}{\longrightarrow} k[S] \rightarrow 0 .
$$

For any $m \in S$ (or even $m \in G(S)$ ) we define (inspired in some graphs of [47]) the simplicial complex :

$$
\Delta_{m}=\left\{F \subset \Lambda \mid m-n_{F} \in S\right\}
$$

where $n_{F}=\sum_{m^{\prime} \in F} m^{\prime}$. Let $\widetilde{H}_{i}\left(\Delta_{m}\right)$ be the $k$-vector space of the $i$ th reduced homology of $\Delta_{m}$, and $\widetilde{h}_{i}\left(\Delta_{m}\right)=\operatorname{dim}\left(\widetilde{H}_{i}\left(\Delta_{m}\right)\right)$.

There exists an effective isomorphism

$$
\text { (*) } \widetilde{H}_{i}\left(\Delta_{m}\right) \simeq V_{i}(m)
$$

for any $m \in S$ and for any $i, 1 \leq i \leq n-2$, (for details see [14],[16] and [7], or also [1]). These isomorphisms are a bridge between Combinatorics and Algebra. For example, notice that the numbers $b_{i}$ in the long resolution can be described by the following formula

$$
b_{i+1}=\sum_{m \in S} \widetilde{h}_{i}\left(\Delta_{m}\right)
$$

Another example, $k[S]$ is Cohen Macaulay if and only if one has $\widetilde{H}_{n-d}\left(\Delta_{m}\right)=0$ for every $m \in S$, where $d=\operatorname{rank} G(S)$. If $k[S]$ is Cohen Macaulay then the Cohen Macaulay type $\tau_{k[\mathbf{X}]}$ of $k[S]$ is given by

$$
\tau_{k[\mathbf{X}]}=\sum_{m \in S} \widetilde{h}_{n-d-1}\left(\Delta_{m}\right)
$$

Thus, in particular, $k[S]$ is Gorenstein if and only if $k[S]$ is Cohen Macaulay and if $\widetilde{H}_{n-d-1}\left(\Delta_{m}\right) \neq 0$ exactly for one $m$ for which, moreover, one has $\widetilde{h}_{n-d-1}\left(\Delta_{m}\right)=1$. The formula for $\tau_{k[\mathbf{X}]}$ follows from the fact that $\tau_{k[\mathbf{X}]}=b_{n-d}$ in the Cohen Macaulay case. Moreover, it is possible to generalize the well known characterization of Gorensteiness for numerical semigroups due to Kunz [32]. To state the result, notice that for $m \in G(S)-S, \Delta_{m}$ is the empty simplicial complex and therefore one has $\widetilde{H}_{i}\left(\Delta_{m}\right)=0$ for such an $m$ and $i \geq-1$. Also notice that $\Delta_{0}$ is the only complex among the $\Delta_{m}$ 's with the property $\widetilde{H}_{-1}\left(\Delta_{m}\right) \neq 0$ (in fact it is a one dimensional space). Finally set $\widetilde{H}_{i}\left(\Delta_{m}\right)=0$ for $i \in \mathbb{Z}, i<-1$, and $m \in G(S)$. From the symmetry 
of the graded resolution in the Gorenstein case, if $R$ is Gorenstein and let $m \in S$ be the element such that $\widetilde{H}_{n-d-1}\left(\Delta_{m}\right) \neq 0$, then for any couple of elements $m_{1}, m_{2} \in G(S)$ with $m_{1}+m_{2}=m$ and $i \in \mathbb{Z}$ one has

$$
\widetilde{H}_{i}\left(\Delta_{m_{1}}\right) \simeq \widetilde{H}_{n-d-i-2}\left(\Delta_{m_{2}}\right)
$$

(see [7] for details).

In the case of a numerical semigroup, let $c$ be the least element, such that $m \in S$ for any $m \geq c$. $\widetilde{H}_{i}\left(\Delta_{m}\right)=0$ for any $m \geq c+n_{\Lambda}-1$ and any $i$, because $\Delta_{m}$ is the full simplex. Therefore, if $S$ is symmetric, the above isomorphism provides a symmetric property on the matrix $\left\{\widetilde{h}_{i}\left(\Delta_{m}\right)\right\}_{i, m}$. (This particular case was proved in [14].)

Another important application of these isomorphisms is the construction of minimal generating sets of syzygies. Notice that

$$
S(i):=\left\{m \in S \quad \mid \widetilde{H}_{i}\left(\Delta_{m}\right) \neq 0\right\}, n-2 \geq i \geq 0,
$$

is the set of $S$-degrees for the minimal $i$-syzygies. The notherian property guarantees that $S(i)$ is a finite set, therefore the following construction provides a method for computing a minimal generating set of $N_{i}$.

\section{Construction:}

Step 1: Compute $S(i)$.

Step 2: For any $m \in S(i)$, take the images of the elements in a basis for the $i$ th reduced homology space $\widetilde{H}_{i}\left(\Delta_{m}\right)$ by the isomorphism.

Step 1 is completely solved in [12], but the partial solution for $i=0$ appears in [8], and for $i=1$ in [43]. Step 2 is solved with an algorithmic method in [7, Remark 3.6].

The case $i=0$ corresponds to the ideal $I=N_{0}$. In this case, step 1 is equivalent to determine the element $m \in S$ such that $\Delta_{m}$ is non-connected. These elements are characterized by the concept of to be m-isolated ([16]) given by three arithmetical conditions. Concretely:

Let $m \in S$, and let $B=\left\{i_{1}, \ldots, i_{p}\right\} \subset C \subset \Lambda, C \neq \Lambda$. We shall say $B$ is $m$-isolated from $\Lambda-C$ if:

1. It is possible to write

$$
m=\sum_{j=1}^{p} \gamma_{i_{j}} n_{i_{j}}=\sum_{t \notin C} \rho_{t} n_{t},
$$

where $\gamma_{i_{j}}, \rho_{t} \in \mathbb{N}$, and $0<\gamma_{i_{j}}$ for any $j, 1 \leq j \leq p$. 
2. If there exists $m^{\prime} \in S$ such that it is possible to write

$$
m^{\prime}=\sum_{j=1}^{p} \gamma_{i_{j}}^{\prime} n_{i_{j}}=\sum_{t \notin B} \rho_{t} n_{t},
$$

with $\gamma_{i_{j}}^{\prime}, \rho_{t} \in \mathbb{N}, \gamma_{i_{j}}^{\prime} \neq 0$, and there exists $t \notin C$ such that $\rho_{t} \neq 0$, then

$$
\left(\gamma_{i_{1}}^{\prime}, \ldots, \gamma_{i_{p}}^{\prime}\right) \nless\left(\gamma_{i_{1}}, \ldots, \gamma_{i_{p}}\right) .
$$

3. If $B^{\prime}=\left\{l_{1}, \ldots, l_{s}\right\} \subset B$ and there exists $m^{\prime} \in S$ such that it is possible to write

$$
m^{\prime}=\sum_{j=1}^{s} \gamma_{l_{j}}^{\prime} n_{l_{j}}=\sum_{t \notin B^{\prime}} \rho_{t} n_{t},
$$

with $\gamma_{l_{j}}^{\prime}, \rho_{t} \in \mathbb{N}$, and there exists $t \notin C$ such that $\rho_{t} \neq 0$, then

$$
\left(\gamma_{l_{1}}^{\prime}, \ldots, \gamma_{l_{s}}^{\prime}\right) \not \subset\left(\gamma_{l_{1}}, \ldots, \gamma_{l_{s}}\right) \text {. }
$$

We obtain the following result:

Theorem 1 ([16]) Let $m \in S$, the following conditions are equivalents:

1: $\Delta_{m}$ is non-connected $\left(\widetilde{H}_{0}\left(\Delta_{m}\right) \neq 0\right)$.

2: There exists $C \subset \Lambda$, such that:

$-C=\cup_{j=1}^{g} T_{j}$.

$-T_{j}$ is $m$-isolated from $\Lambda-C$, for any $j$.

$-T_{j} \cap T_{j+1} \neq \emptyset$, for any $j, 1 \leq j \leq g-1$.

This characterization allows us to find the particular solutions given for few generators in the numerical case in [29] $(n=3),[6]$ and [40] $(n=4)$, and [15] $(n=5)$. Moreover, by means of new combinatorial elements, the theorem yields an algorithm. Concretely, the vertices of some ladders, or equivalently, the Hilbert bases of some diophantine systems are used. (See [8] for details).

The case $i=1$ is solved in [43] by construction of a finite set containing $S(1)$. This set is obtained after studying the non-null spaces $\widetilde{H}_{1}\left(\Delta_{m}\right) \neq(0)$. The concept of $F$-cavity in $\Delta_{m}$ allows us to associate with $S$ some diophantine systems. The Hilbert bases of these systems provide a check finite set. This technique is generalized in [12] for $i \geq 2$. A new concept is necessary, the $i$-triangulation in $\Delta_{m}$.

Now, consider the $S$-graded minimal free resolution of $k[S]$ as $k\left[\mathbf{X}_{E}\right]$ module

$$
0 \rightarrow k\left[\mathbf{X}_{E}\right]^{\beta_{f-1}} \stackrel{\Phi_{f-1}}{\longrightarrow} \cdots \rightarrow k\left[\mathbf{X}_{E}\right]^{\beta_{2}} \stackrel{\Phi_{2}}{\longrightarrow} k\left[\mathbf{X}_{E}\right]^{\beta_{1}} \stackrel{\Phi_{1}}{\longrightarrow} k\left[\mathbf{X}_{E}\right]^{\beta_{0}} \stackrel{\Phi_{0}}{\longrightarrow} k[S] \rightarrow 0 .
$$


This resolution can be described by means of other simplicial complexes ([13]). Concretely, if $m \in S$, let $T_{m}$ be the simplicial complex

$$
T_{m}=\left\{F \subset E \mid m-n_{F} \in S\right\} .
$$

Denote $\widetilde{H}_{i}\left(T_{m}\right)$ the $i$ th reduced homology space of the simplicial complex $T_{m}$, and let $\widetilde{h}_{i}\left(T_{m}\right)=\operatorname{dim}\left(\widetilde{H}_{i}\left(T_{m}\right)\right)$. There exists an isomorphism

$$
\text { (**) } \quad \widetilde{H}_{i}\left(T_{m}\right) \simeq W_{i}(m),
$$

for any $m \in S$ and for any $i, 1 \leq i \leq f-2$ (see [41]). As an application of these isomorphisms, if denote

$$
D(i):=\left\{m \in S \quad \mid \widetilde{H}_{i}\left(T_{m}\right) \neq 0\right\},
$$

we obtain that

$$
\beta_{i+1}=\sum_{m \in D(i)} \widetilde{h}_{i}\left(T_{m}\right), 0 \leq i \leq f-2 .
$$

Notice that, by the noetherian property, $D(i)$ is finite.

In [10] is shown how the sets $D(i)$ can be obtained generalizing the techniques used for computing $S(i)$ in [12]. This process will be recalled in the following section.

Let $A=\Lambda \backslash E, \sharp A=n-f=r$. A first application of the above formula is that $S(i) \subset C_{i}$, where

$$
\begin{array}{r}
C_{i}=\left\{m \in S \mid m=\bar{m}+n_{F}, \text { with } \bar{m} \in D(t) \text { and } F \subset A, \sharp F=i-t,\right. \\
\text { for some } t \geq-1\}
\end{array}
$$

(see [13]). Therefore, in order to determine the set $S(i)$ it is enough to compute $D(t)$ for any $t,-1 \leq t \leq \min (i, f-2)$. Notice that this result allows us to construct the long resolution from the short one.

\section{3. $i$-Triangulations}

The objective of this section is to describe how the sets $S(i), 0 \leq i \leq n-2$, and $D(i),-1 \leq i \leq f-2$, can be obtained solving diophantine systems in non negative integers.

Notice that $D(-1)=Q$, and since $C(E)=C(S)$ for any element $a \in A$ there exists $q_{a} \in \mathbb{N}$ such that $q_{a} \cdot a=\sum_{e \in E} \lambda_{e} \cdot e$ with $\lambda_{e} \in \mathbb{N}$.

Remark 1 Therefore, in order to obtain the set $Q$, one can do:

1. Compute the bounds $q_{a}, a \in A$.

2. Determine the elements $m=\sum_{a \in A} \lambda_{a} \cdot a$, with $\lambda_{a} \in \mathbb{N}$ and $\lambda_{a}<q_{a}$.

3. Check whether the elements $m$ are in $Q$ using Integer Linear Programming. 
Notice that one can compute the set $Q$ solving some diophantine equations in non negative integers, although this way is not practical.

For solving the other cases, we need the concept of $i$-triangulation in a simplicial complex. Let $\Delta$ be an abstract simplicial complex with vertices over a finite set $\mathcal{V}$.

The reduced $i$-homology of the simplicial complex $\Delta$ is the $k$-vector space

$$
\widetilde{H}_{i}(\Delta)=\widetilde{Z}_{i}(\Delta) / \widetilde{B}_{i}(\Delta),
$$

where $\widetilde{Z}_{i}(\Delta)$ and $\widetilde{B}_{i}(\Delta)$ are the spaces of cycles and boundaries respectively.

Let $i \geq 0$ and $F \subset \mathcal{V}$. We will say that $\tau=\left\{F_{1}, \ldots, F_{t}\right\}$ is an $i$-triangulation of $F$ if the following properties are satisfied:

1. $\sharp F_{j}=i+1, \forall j=1, \ldots, t$.

2. $F=\bigcup_{j=1}^{t} F_{j}$.

We will say that $\tau$ is an $i$-triangulation of $F$ in $\Delta$, if $F_{j} \in \Delta, \forall j=1, \ldots, t$, and $F \notin \Delta$.

If $\widetilde{H}_{i}(\Delta) \neq 0$, then there is $c \in \widetilde{Z}_{i}(\Delta)-\widetilde{B}_{i}(\Delta), c=\sum_{j=1}^{t} \lambda_{j} F_{j}$, such that $\tau=\left\{F_{1}, \ldots, F_{t}\right\}$ is an $i$-triangulation of $F$ in $\Delta$, for $F=\bigcup_{j=1}^{t} F_{j}$.

In the cases $\Delta=\Delta_{m}$ or $T_{m}, \mathcal{V}=\Lambda$ or $E$ respectively, if $F \subset \mathcal{V}$, and $\tau=\left\{F_{1}, \ldots, F_{t}\right\}$ is an $i$-triangulation of $F$, in $\Delta_{m}$ or respectively in $T_{m}$, we can associate with $\tau$ a diophantine system solution. Concretely, let $\mathcal{G}$ be the matrix whose columns are the chosen generators of $S, \mathcal{G}:=\left(m_{1}|\ldots| m_{n}\right) \in$ $\mathcal{M}_{(d+s) \times n}(\mathbb{Z})$, considering the $m_{i}$ as elements in $\mathbb{Z}^{d+s}$, and let

$$
\mathcal{G}(t):=\left(\begin{array}{rrrrrrrr}
\mathcal{G} & -\mathcal{G} & 0 & 0 & 0 & & 0 & 0 \\
0 & \mathcal{G} & -\mathcal{G} & 0 & 0 & \cdots & 0 & 0 \\
0 & 0 & \mathcal{G} & -\mathcal{G} & 0 & & 0 & 0 \\
& & \ddots & \ddots & \ddots & & & \\
0 & 0 & 0 & 0 & 0 & & \mathcal{G} & -\mathcal{G}
\end{array}\right) \in \mathcal{M}_{(d+s)(t-1) \times n t}(\mathbb{Z}) .
$$

Let $e_{F_{l}} \in \mathbb{N}^{n}$ be the vector with all its coordinates zero except those indicated in the set $F_{l}$. Let $e_{\tau}:=\left(e_{F_{1}}, \ldots, e_{F_{t}}\right) \in \mathbb{N}^{n t}$ and let

$$
R_{\tau}:=\left\{\alpha=\left(\alpha^{(1)}, \ldots, \alpha^{(t)}\right) \in \mathbb{N}^{n t} \mid \mathcal{G}(t) \alpha=0, \alpha \gg e_{\tau}\right\},
$$

where $\gg$ is the natural partial order in $\mathbb{N}^{n t}$. Since $\tau$ is a $i$-triangulation of $F$ in $\Delta_{m}$ (respectively $T_{m}$ ), there is $\alpha \in R_{\tau}$ such that $\mathcal{G} \alpha^{(1)}=\cdots=\mathcal{G} \alpha^{(t)}=$ $m \in S$.

Notice that $R_{\tau}$ doesn't depend on $m$. Therefore, given $F \subset \mathcal{V}$, and $\tau=\left\{F_{1}, \ldots, F_{t}\right\}$ a $i$-triangulation of $F$, we can consider the set $R_{\tau}$ and the set

$$
\Sigma R_{\tau}:=\left\{m \in S \mid m=\mathcal{G} \alpha^{(1)} \text {, for some } \alpha=\left(\alpha^{(1)}, \alpha^{(2)}, \ldots, \alpha^{(t)}\right) \in R_{\tau}\right\} .
$$


The elements $m \in S$ such that $\tau$ is $i$-triangulation of $F$ in $\Delta_{m}$ or respectively in $T_{m}$ are in $\Sigma R_{\tau}$. We need to be more precise and to give a finite subset of $\Sigma R_{\tau}$ with the same property. For this, we consider

$$
\mathcal{H} R_{\tau}:=\left\{\alpha \in R_{\tau} \mid \alpha \text { is minimal for } \ll\right\},
$$

which is finite, and

$\Sigma \mathcal{H} R_{\tau}:=\left\{m \in S \mid m=\mathcal{G} \alpha^{(1)}\right.$, for any $\left.\alpha=\left(\alpha^{(1)}, \alpha^{(2)}, \ldots, \alpha^{(t)}\right) \in \mathcal{H} R_{\tau}\right\}$.

In the case of the complexes $\Delta_{m}$, one has that ([42])

$$
S(i) \subset \bigcup_{F \subset \Lambda, \sharp F \geq i+2} \bigcup_{\tau} \Sigma \mathcal{H} R_{\tau} .
$$

In the case of the complexes $T_{m}$, we also need to consider the set $Q$. In both cases, there is a partial order which refines the final result. The order is different in each case. Concretely:

- $m>_{S} m^{\prime}$ if $m-m^{\prime} \in S$.

- $m>_{Q} m^{\prime}$ if $m-m^{\prime} \in S \backslash Q$.

Moreover, if $H \subset S$, we will say that $m \in H$ is $S$-minimal (resp. $Q$-minimal) in $H$ if $m \geq_{S} m^{\prime}$ (resp. $m \geq_{Q} m^{\prime}$ ), with $m^{\prime} \in H$, implies that $m=m^{\prime}$.

Let

$$
C_{\tau}:=\left\{m \in \Sigma R_{\tau} \mid m \text { is } S \text {-minimal in } \Sigma R_{\tau}\right\},
$$

and $M_{\tau}:=\left\{m \in \Sigma R_{\tau} \mid m\right.$ is $Q$-minimal in $\left.\Sigma R_{\tau}\right\}$.

$C_{\tau}$ is finite because $C_{\tau} \subset \Sigma \mathcal{H} R_{\tau}$ (see [12] for details). $M_{\tau}$ is finite because $M_{\tau} \subset \Sigma \mathcal{H} R_{\tau}+Q$ (see [10] for details).

The relation of the elements in $S(i)$ (resp. $D(i)$ ) and $C_{\tau}$ (resp. $M_{\tau}$ ) is the following: If $m \in S(i)$ (resp. $m \in D(i)$ ), then there exists $\tau=\left\{F_{1}, \ldots, F_{t}\right\}$, $i$-triangulation of $F=\cup_{j=1, \ldots, t} F_{j}$, such that $m \in C_{\tau}$ (resp. $m \in M_{\tau}$ ). (See [12] and resp. [10] for details.)

Thus, if

$$
C_{\tau}^{\prime}:=\left\{m \in C_{\tau} \mid F \notin \Delta_{m}\right\}, \text { and } C_{i}(F):=\bigcup_{\tau} C_{\tau}^{\prime},
$$

and if

$$
M_{\tau}^{\prime}:=\left\{m \in M_{\tau} \mid F \notin T_{m}\right\}, \text { and } M_{i}(F):=\bigcup_{\tau} M_{\tau}^{\prime},
$$

we obtain the following theorem which provides an algorithm to compute the sets $S(i)$ and $D(i)$ (see [12] and [10] for details). 
Theorem 2 The elements $m \in S$ such that the simplicial complex $\Delta_{m}$, respectively $T_{m}$, has ith reduced homology non null can be determined using Integer Linear Programming. Concretely,

- $S(i) \subset \bigcup_{F \subset \Lambda, \sharp F \geq i+2} C_{i}(F), 0 \leq i \leq n-2$.

- $D(i) \subset \bigcup_{F \subset E, \sharp F \geq i+2} M_{i}(F), 0 \leq i \leq f-2$.

Notice that the integer programming problem for computing the sets $S(i)$ and $D(i)$ can be solved determining the Hilbert bases of some diophantine systems. It is clear that this is not a practical method. Nevertheless, since any element in the Hilbert basis can be bounded in function of the associated matrix, we obtain an explicit bound for the degree of the $i$ th minimal syzygies.

Proposition 3 ([11]) Let $m \in S$ be the degree of a ith minimal syzygy, $0 \leq i \leq n-2$. There is $x$ such that $m=\mathcal{G} x$ with

$$
\begin{aligned}
\|x\|_{1} \leq r & \left(1+2 \max \left|a_{j}\right|+\|\mathcal{G}\|\right)^{(d+s)} \\
& +\left(1+2 \max \left|a_{j}\right|+4\|\mathcal{G}\|\right)^{(d+s)(c-1)}+(i+1)(c+1)-1,
\end{aligned}
$$

where $c=\left(\begin{array}{c}f \\ \lfloor f / 2\rfloor\end{array}\right)$, and $\|\mathcal{G}\|:=\sup _{l} \sum_{j}\left|g_{l j}\right|$.

Other applications are obtained in the case of the ideal $I$ is homogeneous for the natural grading. This case is called projective case, because the ideal $I$ defines a toric projective variety (see for example [50]).

A characterization of when the ideal $I$ is homogeneous is the following:

Proposition 4 ([10]) I is homogeneous for the natural grading if and only if there exists $\mathbf{w} \in \mathbb{Q}^{d}$ such that $\mathbf{w} \cdot \pi\left(m_{i}\right)=1$, for any $i=1, \ldots, n$.

Here we are supposing that

$$
G(S)=\mathbb{Z}^{d} \oplus \mathbb{Z} / a_{1} \mathbb{Z} \oplus \cdots \oplus \mathbb{Z} / a_{s} \mathbb{Z},
$$

with $a_{i} \in \mathbb{Z}$ non null, $1 \leq i \leq s, n=d+s$, and that $\pi$ is the projection over the first coordinates

$$
\pi: \mathbb{Z}^{d+s} \longrightarrow \mathbb{Z}^{d}
$$

Assume that $I$ is a homogeneous ideal. In this case, it is well defined $\|m\|=\|\alpha\|_{1}$, where $m=\sum_{i=1}^{n} \alpha_{i} m_{i}$ and $\|\alpha\|_{1}=\sum_{i=1}^{n} \alpha_{i}$. 
It is well-known (see, for example, [4]) that the regularity of $I$ is

$$
\operatorname{reg}(I)=\max _{0 \leq i \leq n-2}\left\{t_{i}-i\right\}
$$

where $t_{i}$ is the maximum degree of the minimal $i$-syzygies of $I$, i.e.

$$
t_{i}=\max \{\|m\| \mid m \in S(i)\} .
$$

Moreover, the following formula is obtained.

Theorem 5 ([10])

$$
\operatorname{reg}(I)=\max _{-1 \leq i \leq f-2}\left\{u_{i}-i\right\}, \quad \text { where } \quad u_{i}=\max \{\|m\| \mid m \in D(i)\} .
$$

Therefore, one can read the regularity in the short resolution, it is not necessary to use the large one. We obtain an explicit bound for the regularity.

Theorem 6 ([11])

$$
\begin{aligned}
\operatorname{reg}(I) \leq r & \left(1+2 \max \left|a_{j}\right|+\|\mathcal{G}\|\right)^{(d+s)} \\
& +\left(1+2 \max \left|a_{j}\right|+4\|\mathcal{G}\|\right)^{(d+s)(c-1)}+(f-1)(c-1) .
\end{aligned}
$$

Notice that this bound is singly-exponential in the number of extremal rays. Therefore, they are an improvement of the well-known singly-exponential in the number of generators given in [51].

Using again combinatorial techniques, in [45] a new method for computing the minimal generating set of a Lawrence ideal is given. Therefore, a new method for computing Graver bases is obtained. Moreover, a combinatorial characterization of the minimal degrees of a Lawrence ideal is given as well as a degree bound for its minimal first syzygies.

Another result obtained using these techniques is an effective upper bound for the degrees of the equations defining toric projective varieties. Concretely, let $L: S \rightarrow \mathbb{N}$ be the map defined by $L(m)=\|m\|$. For any $t \geq 0$, let $H^{t}:=\{m \in S \mid L(m)=t\}$, and denote

$$
Q^{t}:=Q \cap H^{t}, \quad \text { and } \quad t_{0}:=\min \left\{t \mid Q^{t}=\emptyset\right\} .
$$

On the other hand, from the above sequences, making substitutions of the formulas of homology, the following map is obtained

$$
\bigoplus_{a \in A} \widetilde{H}_{0}\left(T_{m-a}\right) \stackrel{\varphi_{m}}{\longrightarrow} \widetilde{H}_{0}\left(T_{m}\right) \text {. }
$$

Let $t_{1}:=\min \left\{t \mid \operatorname{coker}\left(\varphi_{m}\right)=0 \quad \forall m \in H^{t}\right\}$, i.e. the minimum $t \in \mathbb{N}$ such that $\varphi_{m}$ is surjective for every $m \in H^{t}$.

Theorem 7 ([9]) An effective upper bound for the degrees of the polynomials in a minimal generating set of the equations of a toric projective variety is $\max \left(t_{0}, t_{1}\right)$. 


\section{Computing with Gröbner Bases}

As was explained in section 2, the method proposed in section 3 for computing the sets $S(i)$, allows us to obtain the minimal free resolution of $k[S]$ as $k[\mathbf{X}]$-module, which we have called the long resolution. In [53] are some explicit examples.

Using the sets $C_{i}$ defined in section 2 , it is possible to change slightly the method of the computation of the sets $S(i)$ from the sets $D(t)$ with $-1 \leq t \leq i$ (see [10] for details). The advantage of this change appears when the cardinality of $E$ is strictly less than the cardinality of $\Lambda$. In this case, the number of diophantine systems which one must solve decreases. However, even with this improvement, the obtained method is not faster than the method which employs Gröbner bases.

To compute the long resolution of $k[S]$ using Gröbner bases, one must begin computing the ideal $I$. There exist several methods to do this. Two of them, [22] and [31], are an improvement on the usual method using Elimination Theory (see [50]). These papers consider only the torsion free case. The generalization to non trivial torsion appears in [52]. The advantage of these methods is that they do not need to add new variables like the Elimination theory requires for this concrete problem. An application of these methods is the computation of Hilbert bases of diophantine equations (see [44]), even in the case of equations with congruences (see [43]).

Once a generating set $\left\{f_{1}, \ldots, f_{r}\right\}$ of $I$ is obtained, we can consider the morphism of free $k[\mathbf{X}]$-modules

$$
\varphi: k[\mathbf{X}]^{r} \longrightarrow k[\mathbf{X}]
$$

defined by $\varphi\left(e_{i}\right)=f_{i}$. It is enough to use the Schreyer Theorem to obtain the whole resolution. In fact, some Formal Calculus Systems, as MACAULAY2 or Singular, have installed this algorithm, even with some improvements (see [33]). From the long resolution one can read the sets $S(i)$.

In order to compute the $S$-graded minimal free resolution of $k[S]$ as $k\left[\mathbf{X}_{E}\right]$-module with Gröbner bases, we must begin by computing the Apery set, $Q$. For details see [41].

Assume, for the sake of simplicity,

$$
E=\left\{m_{1}, \ldots, m_{f}\right\} \quad \text { and } \quad A=\left\{m_{f+1}, \ldots, m_{n}\right\} .
$$

Fix a total order on the monomials of $k[\mathbf{X}]=k\left[\mathbf{X}_{E}, \mathbf{X}_{A}\right], X_{1}<X_{2}<\cdots<X_{n}$, such that:

1. $\mathbf{X}^{\alpha}<\mathbf{X}^{\beta}$, implies $\mathbf{X}^{\alpha+\gamma}<\mathbf{X}^{\beta+\gamma}$, for any $\alpha, \beta$ and $\gamma$; 
2. If $f=\sum a_{\alpha} \mathbf{X}^{\alpha} \in k[\mathbf{X}]$ has the leading monomial $\mathbf{X}^{\beta} \notin k\left[\mathbf{X}_{A}\right]$, then $\mathbf{X}^{\alpha} \notin k\left[\mathbf{X}_{A}\right]$, for any $\alpha$ with $a_{\alpha} \neq 0$.

For example, we can consider the lex - inf order, which is defined

$$
\alpha>_{\text {lex-inf }} \beta \Longleftrightarrow \alpha<_{\text {lex }} \beta,
$$

where lex order is the lexicografic order for $X_{1}>\cdots>X_{n}$.

Every order with these properties is not a well-ordering. However, since there exists only a finite number of monomials of $S$-degree $m \in S$, a Gröbner basis of $I$ can be computed from any $S$-graded generating set of $I$.

Assume that $\Gamma$ is the reduced Gröbner basis of $I$ for such an order. Let $\mathcal{B}$ be the set of monomials $\mathbf{X}_{A}^{\alpha}$ which are not divisible by any leading monomial of $\Gamma$.

Lemma $8([41])$

$$
Q=\left\{m \in S \mid m=\sum_{i=f+1}^{n} \alpha_{i} m_{i}, \text { where } \mathbf{X}_{A}^{\alpha} \in \mathcal{B}\right\}
$$

and in particular, the set $\mathcal{B}$ is finite.

Notice that the integer linear problem of computing $Q$ given in Remark 1 is now solved by means of the reduced Gröbner basis of $I$.

Let $l_{0}$ be the cardinality of $\mathcal{B}=\left\{\mathbf{X}_{A}^{\alpha_{1}}, \ldots, \mathbf{X}_{A}^{\alpha_{l_{0}}}\right\}$. Any element in $\Gamma$ whose leading monomial $\mathbf{X}_{E}^{v} \mathbf{X}_{A}^{u}$ has variables in $\left\{X_{i} \mid 1 \leq i \leq f\right\}$ (i.e. $v \neq 0$ ), is, except sign \pm ,

$$
\mathbf{X}_{E}^{v} \mathbf{X}_{A}^{u}-\mathbf{X}_{E}^{v^{\prime}} \mathbf{X}_{A}^{u^{\prime}}
$$

Property 2 of the order says that $v^{\prime} \neq 0$, and therefore, since $\Gamma$ is a reduced Gröbner basis, $\mathbf{X}_{A}^{u}$ and $\mathbf{X}_{A}^{u^{\prime}} \in \mathcal{B}$.

Moreover, $u \neq u^{\prime}$ because otherwise, since $I$ is a saturated ideal, $\mathbf{X}_{E}^{v}-$ $\mathbf{X}_{E}^{v^{\prime}} \in I$, which is a contradiction with $\Gamma$ is a Gröbner basis.

Suppose that $\mathbf{X}_{A}^{u}=\mathbf{X}_{A}^{\alpha_{i}}$, and $\mathbf{X}_{A}^{u^{\prime}}=\mathbf{X}_{A}^{\alpha_{j}}$. For any $\gamma \in \mathbb{N}^{r}$ such that

$$
\mathbf{X}_{A}^{\gamma+\alpha_{i}} \in \mathcal{B} \quad \text { and } \quad \mathbf{X}_{A}^{\gamma+\alpha_{j}} \in \mathcal{B}
$$

if $\alpha_{i^{\prime}}=\gamma+\alpha_{i}$ and $\alpha_{j^{\prime}}=\gamma+\alpha_{j}$, we associate with the chosen element in $\Gamma$, the element in $k\left[\mathbf{X}_{E}\right]^{l_{0}}$ with all the coordinates equal to zero, except the $i^{\prime}$ th and $j^{\prime}$ th ones, which are $\mathbf{X}_{E}^{v}$, and $-\mathbf{X}_{E}^{v^{\prime}}$, respectively. Notice that there is at least an element associated, the one corresponding to $\gamma=0$. 
For any $\gamma \in \mathbb{N}^{r}$ such that

$$
\mathbf{X}_{A}^{\gamma+\alpha_{i}} \in \mathcal{B} \quad \text { and } \quad \mathbf{X}_{A}^{\gamma+\alpha_{j}} \notin \mathcal{B}
$$

if $\alpha_{i^{\prime}}=\gamma+\alpha_{i}$ and $\mathbf{X}_{E}^{w} \mathbf{X}_{A}^{\alpha_{j^{\prime}}}$ is the division remainder of $\mathbf{X}_{A}^{\gamma+\alpha_{j}}$ by $\Gamma$, we associate with the chosen element in $\Gamma$, the element in $k\left[\mathbf{X}_{E}\right]^{l_{0}}$ with all the coordinates equal to zero, except the $i^{\prime}$ th and $j^{\prime}$ th ones, which are $\mathbf{X}_{E}^{v}$, and $-\mathbf{X}_{E}^{v^{\prime}+w}$, respectively.

In this way, taking all the elements in $\Gamma$ of the above form, we obtain $G_{i} \in k\left[\mathbf{X}_{E}\right]^{l_{0}}, 1 \leq i \leq l_{1}$.

Notice that $\beta_{0} \leq l_{0}$. In the case $\beta_{0}<l_{0}$, if $\beta_{0}+1 \leq i \leq l_{0}, q_{i}=q_{j}$ for a unique $j, 1 \leq j \leq \beta_{0}$. Denote $j=j(i)$. We consider

$$
\pi: k\left[\mathbf{X}_{E}\right]^{l_{0}} \rightarrow k\left[\mathbf{X}_{E}\right]^{\beta_{0}},
$$

the $k\left[\mathbf{X}_{E}\right]$-module morphism defined by

$$
\pi\left(e_{i}\right)= \begin{cases}e_{i} & \text { if } 1 \leq i \leq \beta_{0} \\ e_{j(i)} & \text { if } \beta_{0}+1 \leq i \leq l_{0}\end{cases}
$$

We consider the matrix

$$
\mathcal{M}:=\left(\pi\left(G_{1}\right)|\cdots| \pi\left(G_{l_{1}}\right)\right) .
$$

$\mathcal{M}$ defines a morphism of free $k\left[\mathbf{X}_{E}\right]$-modules

$$
\Psi_{1}: k\left[\mathbf{X}_{E}\right]^{l_{1}} \longrightarrow k\left[\mathbf{X}_{E}\right]^{\beta_{0}}
$$

Proposition 9 ([41])

$$
\operatorname{coker}(\mathcal{M}) \simeq_{k\left[\mathbf{x}_{E}\right]} k[S]
$$

Therefore, we obtain the first step of a free resolution of $k[S]$ as $k\left[\mathbf{X}_{E}\right]$ module that is $S$-graded.

$$
k\left[\mathbf{X}_{E}\right]^{l_{1}} \stackrel{\Psi_{1}}{\longrightarrow} k\left[\mathbf{X}_{E}\right]^{\beta_{0}} \stackrel{\Phi_{0}}{\longrightarrow} k[S] \rightarrow 0
$$

Now, in order to obtain the short resolution, it is enough to apply the Schreyer Theorem. The sets $D(i)$ can be read from the short resolution.

We conclude that the integer programming problem for computing $S(i)$ and $D(i)$ given in Theorem 2 can be solved by means of Schreyer Theorem, therefore using Gröbner bases. 


\section{References}

[1] Aramova, A. and Herzog, J.: Free resolution and Koszul homology. J. of Pure Appl. Algebra 105 (1995), no. 1, 1-16.

[2] Bayer, D., Peeva, I. And Sturmfels, B.: Monomial resolutions. Math. Res. Lett. 5, (1998), no. 1-2, 31-46.

[3] Bayer, D., Popescu, S. And Sturmfels, B.: Syzygies of unimodular Lawrence ideals. J. Reine Angew. Math. 534 (2001), 169-186.

[4] Bayer, D. And Stillman, M.: A criterion for detecting m-regularity. Invent. Math. 87 (1987), no. 1, 1-11.

[5] Bayer, D. And Sturmfels, B.: Cellular resolutions of monomial modules. J. Reine Angew. Math. 502 (1998), 123-140.

[6] Bresinsky, H.: Binomial generating sets for monomial curves, with applications in $\mathbb{A}^{4}$. Rend. Sem. Mat. Univ. Politec. Torino 46 (1998), no. 3, 353-370 (1990).

[7] Briales, E., Campillo, A., Marijuán, C. and Pisón, P.: Combinatorics of syzygies for semigroup algebras. Collect. Math. 49, (1998), no. 2-3, $239-256$.

[8] Briales, E., Campillo, A., Marijuán, C. and Pisón, P.: Minimal systems of generators for ideals of semigroups. J. Pure Appl. Algebra 124 (1998), no. 1-3, 7-30.

[9] Briales, E., Campillo, A. and Pisón, P.: On the equations defining toric projective varieties. In Geometric and Combinatorial Aspects of Commutative Algebra (Messina, 1999), 57-66. Lecture Notes in Pure and Appl. Math. 217, Dekker, New York, 2001.

[10] Briales, E., Campillo, A., Pisón, P. and Vigneron, A.: Simplicial complexes and syzygies of lattice ideals. In Symbolic Computation: Solving Equations in Algebra, Geometry and Engineering, 169-183, Contemp. Math. 286, Amer. Math. Soc., Providence, RI, 2001.

[11] Briales, E., Pisón, P. And Vigneron, A.: Cotas para la Regularidad de un Ideal de Retículo. In Actas del Encuentro de Matemáticos Andaluces, 171-178. Servicio de publicaciones de la Univ. de Sevilla, 2002.

[12] Briales, E., Pisón, P. And Vigneron, A.: The Regularity of a Toric Variety. J. Algebra 237 (2001), no. 1, 165-185.

[13] Campillo, A. And Giménez, P.: Syzygies of affine toric varieties. J. Algebra 225 (2000), no. 1, 142-161.

[14] Campillo, A. And Marijuán, C.: Higher order relations for a numerical semigroup. Sém. Théor. Nombres Bordeaux (2) 3 (1991), no. 2, 249-260.

[15] Campillo, A. and Pisón, P.: Generators of a monomial curve and graphs for the associated semigroup. Bull. Soc. Math. Belg. Sér. A 45 (1993), no. $1-2,45-58$.

[16] Campillo, A. And Pisón, P.: L'idéal d'un semi-groupe de type fini. C. R. Acad. Sci. Paris Sér. I Math. 316 (1993), no. 12, 1303-1306. 
[17] Campillo, A. And Pisón, P.: Toric Mathematics from semigroup viewpoint. In Ring Theory and Algebraic Geometry, 95-112. Lectures Notes in Pure and Applied Mathematics 221, Dekker, New York, 2001.

[18] Clausen, M. and Fortenbacher, A.: Efficient solution of linear Diophantine equations. J. Symbolic Comput. 8 (1989), no. 1-2, 201-216.

[19] Conti, P. and Traverso, C.: Buchberger algorithm and integer programming. In Applied algebra, algebraic algorithms and error-correcting codes, New Orleans, LA, 1991, 130-139. Lecture Notes in Comput. Sci., 539, Springer, Berlin, 1991.

[20] Contejean, E. And Devie, H.: An efficient incremental algorithm for solving systems of linear Diophantine equations. Inform. and Comput. 113 (1994), no. 1, 143-172.

[21] Cox, D.: Recent developments in toric geometry. In Algebraic geometry (Santa Cruz, 1995), 389-436. Proc. Sympos. Pure Math. 62, Part 2. Amer. Math. Soc., Providence, RI, 1997.

[22] Di Biase, F. And Urbanke, R.: An algorithm to calculate the kernel of certain polynomial ring homomorphisms. Experiment. Math. 4 (1995), no. $3,227-234$.

[23] Domenjoud, E.: Outils pour la Déduction Automatique dans les Théories Associatives-Commutatives. Thése de doctorat d'Université, Université de Nancy I, 1991.

[24] Eisenbud, D. And Goto, S.: Linear free resolutions and minimal multiplicity. J. Algebra 88 (1984), no. 1, 89-133.

[25] Eisenbud, D. And Sturmfels, B.: Binomial ideals. Duke Math. J. 84 (1996), no. 1, 1-45.

[26] Fulton, W.: Introduction to Toric Varieties. Annals of Mathematics Studies 131. Princeton University Press, Princeton, 1993.

[27] Gelfand, I. Kapranov, M. and Zelevinsky, A.: Discriminants, resultants, and multidimensional determinants. Mathematics: Theory \& Applications, Birkhäuser, Boston, Inc., Boston, MA, 1994.

[28] Graver, J. E.: On the foundations of linear and integer linear programming I. Math. Programming 9 (1975), no. 2, 207-226.

[29] Herzog, J.: Generators and relations of abelian semigroups and semigroup rings. Manuscripta Math. 3 (1970), 175-193.

[30] Hochster, M.: Cohen-Macaulay rings, combinatorics, and simplicial complexes. In Ring theory, II (Proc. Second Conf., Univ. Oklahoma, Norman, Okla., 1975), 171-223. Lect. Notes in Pure and Appl. Math. 26, Dekker, New York, 1977.

[31] Hosten, S. and Sturmfels, B.: GRIN: an implementation of Gröbner bases for integer programming. In Integer programming and combinatorial optimization (Copenhagen, 1995), 267-276. Lecture Notes in Comput. Sci. 920, Springer, Berlin, 1995. 
[32] Kunz, E.: The value-semigroup of a one-dimensional Gorenstein ring. Proc. Amer. Math. Soc. 25 (1970), 748-751.

[33] La Scala, R. and Stillman, M.: Strategies for computing minimal free resolutions. J. Symbolic Comput. 26 (1998), no. 4, 409-431.

[34] OJeda, I. And Piedra, R.: Cellular binomial ideals. Primary decomposition of binomial ideals. J. Symbolic Comput. 30 (2000), no. 4, 383-400.

[35] Ojeda, I. And Piedra, R.: Index of nilpotency of binomial ideals. J. Algebra 255 (2002), no. 1, 135-147.

[36] OJEDA, I. AND PISÓN, P.: The hull resolution of a monomial curve in $\mathbb{A}^{3}(k)$. Prepublicaciones del Departamento de Álgebra de la Universidad de Sevilla 12, 2001.

[37] Papadimitriou, C. H.: On the complexity of integer programming. J. Assoc. Comput. Mach. 28 (1981), no. 4, 765-768.

[38] Peeva, I. And Sturmfels, B.: Generic lattice ideals. J. Amer. Math. Soc. 11 (1998), no. 2, 363-373.

[39] Peeva, I. and Sturmfels, B.: Syzygies of codimension 2 lattice ideals. Math. Z. 229 (1998), no. 1, 163-194.

[40] Pisón, P.: Métodos combinatorios en Álgebra local y Curvas monomiales en dimensión 4. Doctoral thesis, Universidad de Sevilla, 1991.

[41] Pisón, P.: The short resolution of a lattice ideal. Proc. Amer. Math. Soc., to appear.

[42] Pisón-Casares, P. and Vigneron-Tenorio, A.: Computing Toric First Syzygies. International Conference IMACS-ACA, El Escorial, Madrid, Spain, 1999.

[43] Pisón-Casares, P. and Vigneron-Tenorio, A.: First syzygies of toric varieties and Diophantine equations in congruence. Comm. Algebra 29 (2001), no. 4, 1445-1466.

[44] Pisón-Casares, P. and Vigneron-Tenorio, A.: $\mathbb{N}$-solutions to linear systems over $\mathbb{Z}$. Prepublicación de la Universidad de Sevilla, Sección Álgebra 43, 1998.

[45] Pisón-Casares, P. and Vigneron-Tenorio, A.: On the Graver Bases of Semigroup Ideals. Prepublicaciones del Departamento de Álgebra de la Universidad de Sevilla 10 (2001), 1-12, preprint.

[46] Pottier, L.: Minimal solutions of linear diophantine systems: bounds and algorithms. In Rewriting techniques and applications (Como, 1991), 162-173. Lectures Notes in Comput. Sci. 488, Springer, Berlin, 1991.

[47] Rosales, J. C.: Semigrupos numéricos. Doctoral thesis, Universidad de Granada, 1991.

[48] Stanley, R.: Combinatorics and commutative algebra, second edition. Progress in Mathematics 41. Birkhäuser, Boston-Basel-Berlin, 1996.

[49] Sturmfels, B.: Equations defining toric varieties. In Algebraic geometry (Santa Cruz, 1995), 437-449. Proc. Sympos. Pure Math. 62 Part 2, Amer. Math. Soc., Providence, RI, 1997. 
[50] Sturmfels, B.: Gröbner Bases and Convex Polytopes. University Lectures Series 8, American Mathematical Society, Providence, RI, 1995.

[51] Sturmfels, B.: Gröbner bases of Toric Varieties. Tohoku Math. J. (2) 43 (1991), no. 2, 249-261.

[52] Vigneron-Tenorio, A.: Semigroup ideals and linear Diophantine equations. Linear Algebra Appl. 295 (1999), no. 1-3, 133-144.

[53] Vigneron-Tenorio, A.: Álgebras de Semigrupos y Aplicaciones. Doctoral thesis, Universidad de Sevilla, 2000.

Recibido: 8 de abril de 2002

Revisado: 20 de enero de 2003

Emilio Briales-Morales

Departamento de Álgebra

Facultad de Matemáticas, Universidad de Sevilla

Apartado 1160, 41080 Sevilla, Spain

emilio@us.es

Antonio Campillo-López

Departamento de Álgebra, Geometría y Topología

Facultad de Ciencias, Universidad de Valladolid

Prado de la Magdalena, s/n, 47005 Valladolid, Spain

campillo@agt.uva.es

Pilar Pisón-Casares

Departamento de Álgebra

Facultad de Matemáticas, Universidad de Sevilla Apartado 1160, 41080 Sevilla, Spain

ppison@us.es

Alberto Vigneron-Tenorio

Departamento de Matemáticas

EUE Empresariales y de Administración Pública

Universidad de Cádiz

Porvera, 54, 11403 Jerez de la Frontera (Cádiz), Spain

alberto.vigneron@uca.es

Dedicated to Professor J.L. Vicente on his sixtieth birthday. Emilio Briales, Pilar Pisón and Alberto Vigneron are supported by MCyT Spain, BFM2000-1523, and Junta de Andalucía, FQM304. 